\title{
Case Report: Sudden Infant Death Due to Postvaccinal Myocarditis: A Case Report
}

\author{
Babak Salahshour $^{1}$ (D), Sajjad Sadeghi ${ }^{1,2}$ (D), Ahmad Sajadianfar ${ }^{2}$ (D), Kambiz Soltaninejad ${ }^{1 *}$ (C)
}

1. Department of Forensic Toxicology, Legal Medicine Research Center, Legal Medicine Organization, Tehran, Iran.

2. Forensic Pathology Laboratory, Legal Medicine Center of North Khorasan Province, Bojnurd, Iran.

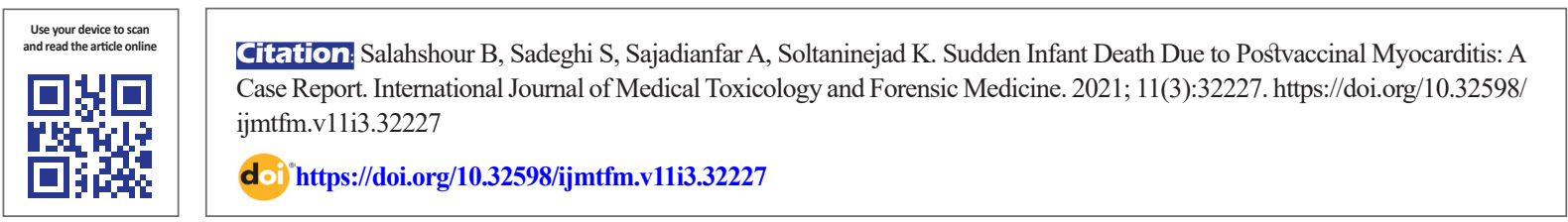

(c) (1) (s)

Article info:

Received: 12 Sep 2020

First Revision: 29 Sep 2020

Accepted: 19 Oct 2020

Published: 25 Aug 2021

\section{Keywords:}

Vaccine, Death, Myocarditis, Histopathology, Case report

\section{ABSTRACT}

Background: Vaccination is a fundamental strategy in public health for controlling and eradicating infectious diseases. However, the undesirable Adverse Events Following Immunization (AEFI) should be effectively monitored, prevented, and managed in societies. Previously, Sudden Infant Death (SID) has been reported as a serious AEFI. We present a rare case of SID in an 18-month-old girl due to severe myocarditis following vaccination.

Case Presentation: An 18-month-old girl presented to the Emergency Department (ED) with a history of the injection of Diphtheria-Tetanus-Whole Cell Pertussis (DTwP); Measles, Mumps, and Rubella (MMR), and oral poliovirus vaccines. She expired one-hour post ED admission after the episodes of vomiting and cardiopulmonary arrest. Postmortem histopathological examination signified marked congestion and the destruction of muscle fibers in the myocardium with inflammatory cells infiltration predominantly eosinophils. Given the postmortem histopathological findings, the cause of death was concluded as severe myocarditis following vaccination. Finally, the causality assessment was performed according to the Naranjo scale; the relevant data revealed a probable association in this regard (Naranjo score 6).

Conclusion: Myocarditis is a rare and probable AEFI; thus, cardiac monitoring should be recommended as a diagnostic measure in managing postvaccinal adverse effects.

\footnotetext{
* Corresponding Author:

Dr. Kambiz Soltaninejad, PharmD, PhD.

Address: Department of Forensic Toxicology, Legal Medicine Research Center, Legal Medicine Organization, Tehran, Iran. Tel: +98 (21) 55613731

E-mail: kamsoltaninejad@gmail.com
} 


\section{Introduction}

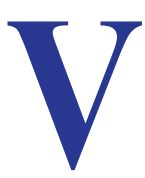

accination is a fundamental and main strategy in public health policy for controlling infectious diseases, especially among infants [1]. In general, vaccination programs successfully led to the eradication of infectious diseases and increased life expectancy. This program is among the most suitable and economic public health measures. Vaccination programs have improved the primary health infrastructure and decreased morbidity and mortality in the public [2]. However, the undesirable adverse events of the vaccines should be monitored in society; they should be effectively prevented and managed based on the reports [3]. Safety monitoring of vaccines used in immunization programs is essential in all countries. However, identification, detection, prevention, and appropriate communication of Adverse Events Following Immunization (AEFI) is essential to preserve the safety of vaccination schedules [4].

Sudden death is a critical issue in forensic medicine. It can be defined as an unexpected death in an individual without any known disease, injury, or disorder within 24 hours after the onset of an illness [5]. Sudden Infant Death (SID) was reported as a serious AEFI [6, 7]. Additionally, the probable association between vaccination and SIDs has been noted. This is because the peak age for the incidence of sudden deaths coincides with the age of primary vaccination [8]. Age, gender, injection site reactions, and the vaccine biological components are the main factors that affect the severity and incidence of vaccine-related adverse effects; thus, the severity and incidence rate of AEFI are different [1].

Here, we reported a rare case of SID due to severe myocarditis following immunization with vaccines in an 18-month-old girl. The presented data were based on forensic autopsy findings and postmortem histopathological examination.

\section{Case Presentation}

An 18-month-old girl was referred by her mother from a rural area and presented to the Emergency Department (ED) of a local hospital (Bojnurd City, North-East of Iran). She reported a history of intramuscular injection of Diphtheria-Tetanus-Whole Cell Pertussis (DTwP) vaccine, the subcutaneous injection of Measles, Mumps, and Rubella (MMR), and Oral Poliovirus Vaccine (OPV) (Razi Vaccine \& Serum Research Institute, Alborz Province, Karaj, Iran) about 48 hours ago, according to routine national immunization program. She encountered episodes of nausea and vomiting with restlessness, crying, and fever after receiving vaccines. In their medical history, she manifested no significant congenital or comorbid medical illnesses. Post-vaccination, she was put on acetaminophen [Oral drop $(100 \mathrm{mg} / \mathrm{mL}), 20$ drops/ every 6 hours] for the management of fever and pain. Thirty minutes before ED admission, she experienced an episode of vomiting and remained unconscious. On admission to the ED, she was in cardiopulmonary arrest status. She was cyanotic with double mydriasis and presented no vital signs. Accordingly, cardio-respiratory arrest, endotracheal intubation, and Cardiopulmonary Resuscitation (CPR) were performed. The deterioration of cardiac function was as ventricular tachycardia and asystole. Despite CPR, she expired due to cardiopulmonary arrest (one-hour post-admission to the ED). The decedent was transferred to forensic medicine center for medicolegal investigation and determining the cause of death.

A forensic autopsy was performed approximately 12 hours after the death. The infant's height was $82 \mathrm{~cm}$ and her weight was $10 \mathrm{~kg}$. Her body presented age-related healthy development. In the external examination, there was no evidence of physical or congenital deformities or putrefaction. No traumatic lesions were observed on the body. There was no evidence of serious injury on the body except external CPR features in the chest and inhospital injection marks due to therapeutic procedures. Both eyes and the face were pale, and petechial hemorrhages were observed in the conjunctivae and sclera. There was no other soft tissue, cervical spine, laryngeal, or skeletal injuries and fractures on the neck. There was no congestion, submucosal injuries, hemorrhages, or erosion on the nasal cavity. The heart weighed $100 \mathrm{~g}$ with left ventricle mild hypertrophy without any valvular or coronary artery abnormalities. There was no food or vomits materials in the trachea and bronchi. The left and right lungs revealed mild congestion. The stomach was empty and the other organs indicated no obvious abnormality except for congestion. The toxicological analysis provided no evidence of drugs/poisons or alcohol.

For histopathological examinations, tissue samples were fixed with phosphate-buffered formalin and embedded in paraffin. Then, $5 \mu \mathrm{m}$ thickness paraffin sections were stained with Hematoxylin-Eosin (H\&E). In the heart, inflammatory cells infiltration in myocardial fibers predominantly eosinophils, marked congestion, and the destruction of muscle fibers in the myocardium were noted. These findings included severe myocarditis (Figures 1 and 2). 
Due to medico-legal death investigations, including clinical history, forensic autopsy, and postmortem histopathological findings, the cause of death was concluded as severe myocarditis following vaccination.

Finally, the causality assessment was performed using the Naranjo scale [9]. The calculated score was 6 ; this value revealed a probable association between vaccinations and severe myocarditis.

\section{Discussion}

Postmortem histopathological examination revealed severe myocarditis after vaccination in our case. To the best of our knowledge, this is the first report of death following DTPw, OPV, and MMR vaccination with confirmation by postmortem histopathological examination in Iran. In sudden and unexpected deaths, autopsy plays a specific and paramount role in determining the cause of death. An unexpected death due to a cardiovascular accident in an individual is usually called Sudden Cardiac Death (SCD) [5]. Coronary heart disease, cardiac dysrhythmias, and cardiomyopathies are the major causes of SCDs [10, 11]. Myocarditis has been reported as a cause of SCD [12-14]. Vaccinations are usually well tolerated. In some previous studies, sudden death following vaccination has been considered [7, 8, 15-17]; however, myocarditis following vaccination has been reported as a rare AEFI and cause of death [18-20]. Majorly, myocarditis has been reported as a rare adverse effect following DTwP and polio vaccination [18-20]. For example, Boc-

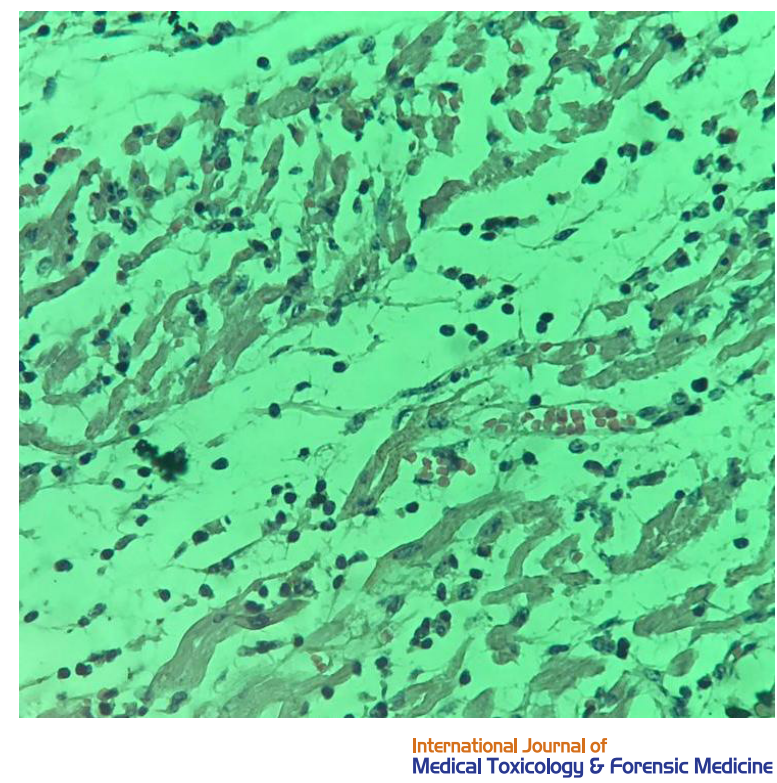

Figure 1. The histopathology of the heart presents myocardial fibers eosinophilic infiltration and marked congestion (H\&E staining $\times 100)$ cara et al. reported the first case of myopericarditis after vaccination against diphtheria, tetanus, and poliovirus in a 31-year-old man. In this case, two days after vaccination, he presented with fever, acute chest pain, and Electrocardiogram (ECG) abnormalities. Myocarditis was confirmed by endomyocardial biopsy and laboratory findings related to the inflammatory syndrome. After treatment with high-dose aspirin, he recovered without any complications [19]. In another case report, acute fulminant myocarditis occurred after immunization in an 8-month-old female after receiving the vaccination against diphtheria, polio, and tetanus. Cardiogenic shock due to extracorporeal membrane oxygenation was a relief and the patient survived; however, she was expired due to heart failure two months later [20].

Kumar et al. [18] published a case report about myocarditis following DTPw vaccination in a 6-week-old female with a large ventricular septal defect. Two days after vaccination, she encountered intermittent lowgrade fever and severe respiratory distress. In physical examination, she was severely tachypneic and tachycardiac with cold peripheries and hypotensive. The paraclinical findings included metabolic acidosis, high erythrocyte sedimentation rate, and C-reactive protein, hypoxia, cardiomegaly, ECG abnormality (sinus tachycardia, nonspecific ST depression), and biventricular dysfunction. Additionally, creatine kinase MB and liver enzymes were elevated. Due to the possibility of postvaccinal myocarditis, along with supportive and symptomatic therapy, steroid administration was initiated and the patient's signs and symptoms settled gradually. The patient was discharged after 4 days [19]. These reports are similar to our observed case. Our reported case presented fever, gastrointestinal presentations, and restlessness after immunization with DTwP, OPV, and MMR vaccines. Furthermore, the case was expired about 28 hours post-vaccination with severe myocarditis. According to reports, myocarditis should be related to the DTwP

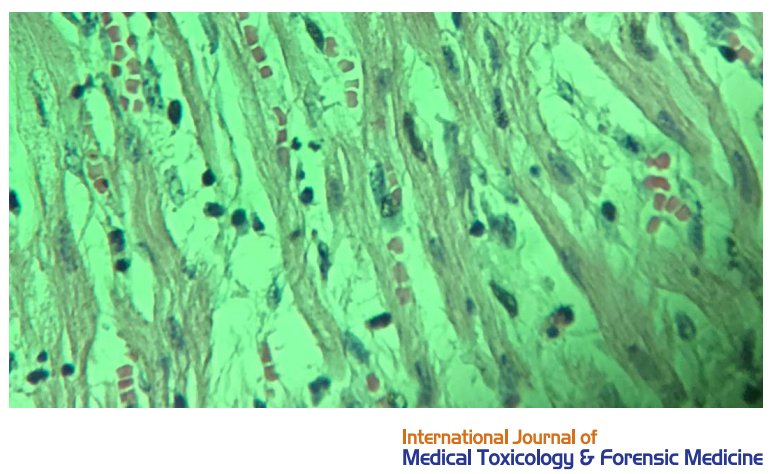

Figure 2. The histopathology of the heart illustrates the myocardial fibers and eosinophilic infiltration (H\&E staining $\times 400)$ 
vaccine. Myocarditis following the DTwP vaccine is very rare. Consistent with previous reports, she had a structurally normal heart.

The proposed mechanism of myocarditis following vaccination is due to immune response dysfunction. The possible mechanisms of myocarditis after vaccination are infective myocarditis and hypersensitivity myocarditis. Infective myocarditis occurs during bacterial, fungal, viral, and protozoal infection. However, in our case, bacteriological and virological tests were not performed. However, there were no associated symptoms of diphtheria, polio, tetanus, and other virus infections in clinical examinations. Hypersensitivity myocarditis is usually related to hypersensitivity reactions to drugrelated immunologic syndromes $[19,20]$. The component of a vaccine causing this immune system dysfunction remains unclear. Studies suggested that the DTwP vaccine, compared to the Diphtheria-Tetanus-Acellular Pertussis (DTaP) vaccine, when administered to populations of similar age and gender, manifests higher adverse events and life-threatening reactions, such as fever, seizures, encephalitis, encephalopathy, and sudden deaths. Whole-Cell pertussis vaccines contain approximately 3000 different proteins, including endotoxin, pertussis toxin, and adenylate cyclase whereas acellular pertussis vaccine contains only $2-5$ proteins $[21,22]$.

Adverse Drug Reaction (ADR) is an unwanted and harmful reaction experienced following the administration of a drug under normal doses used for the prevention, diagnosis, therapy, or modification of disease in humans; it is suspected to be related to the drug [23]. ADRs are crucial causes of morbidity and mortality [23]. Causality assessment is the evaluation of the relationship between a drug and the occurrence of an ADR. Naranjo algorithm or scale is a causality assessment scale. It was developed by Naranjo et al. in 1991 to help standardize the evaluation of causality for all ADRs $[9,23,24]$. The Naranjo scale includes 10 questions. i.e., answered as "Yes", "No", or "Do not know" with different points (-1, $0,+1$, or +2 ) for each answer. Total scores range from -4 to +13 ; the reaction is considered definite if the score is $\geq 9$, probable if between $5-8$, possible if equal to $1-4$, and doubtful if $\leq 0$ [24]. According to this scale, the total score was 6 and suggestive of a probable association between vaccination and severe myocarditis.

To the best of our knowledge, this case is the first report of rare fatal myocarditis following vaccination as a cause of SID with postmortem histopathological evidence in Iran. There exist scant reports from other countries related to postvaccinal myocarditis, due to the difference in vaccine components as biological products with different origins and pharmaceutical brands of vaccines, the immunological-based adverse effects may be different.

The bacteriological and virological tests were not performed for this case; this was among the main limitations of our study. Further studies should be performed to validate our observation and assess the potential of vaccines to cause severe myocarditis.

\section{Conclusion}

This report highlighted a very rare severe myocarditis due to vaccination. In light of the presence of a safer alternative DTwP vaccine, the Iranian health authorities should be switched to using the DTaP vaccine. The present cases emphasize the need for cardiac monitoring during the management of such cases for diagnosis and therapeutic modalities.

\section{Ethical Considerations}

\section{Compliance with ethical guidelines}

The study protocol was in conformity with the ethical guidelines of the 1975 Declaration of Helsinki, revised in 1983. The authors confirm that an informed consent was obtained from the legal relatives of the decedent prior to drafting this report. Private information, including name and surname was removed from the data sheet to comply with ethical concerns.

\section{Funding}

This research received no specific grant from funding agencies in the public, commercial, or non-profit sectors.

\section{Author's contributions}

All authors equally contributed to preparing this article.

\section{Conflict of interest}

The authors declared no conflicts of interest.

\section{Acknowledgments}

We would like to thank the staff of North Khorasan Legal Medicine Center for their kind cooperation. 


\section{References}

[1] Karami M, Ameri P, Bathaei J, Berangi Z, Pashaei T, Zahiri $\mathrm{A}$, et al. Adverse events following immunization with pentavalent vaccine: experiences of newly introduced vaccine in Iran. BMC Immunol. 2017; 18(1):42. [DOI:10.1186/s12865017-0226-8] [PMID] [PMCID]

[2] Andre FE, Booy R, Bock HL, Clemens J, Datta SK, John TJ, et al. Vaccination greatly reduces disease, disability, death and inequity worldwide. Bull World Health Organ. 2008, 86(2):140-6. [DOI:10.2471/BLT.07.040089] [PMID] [PMCID]

[3] Tafuri S, Gallone MS, Calabrese G, Germinario C. Adverse events following immunization: is this time for the use of WHO causality assessment? Expert Rev Vaccines. 2015; 14(5):625-7. [DOI:10.1586/14760584.2015.1029460] [PMID]

[4] Dodoo AN, Renner L, van Grootheest AC, Labadie J, Antwi-Agyei KO, Hayibor S, et al. Safety monitoring of a new pentavalent vaccine in the expanded programme on immunisation in Ghana. Drug Saf. 2007; 30(4):347-56. [DOI:10.2165/00002018-200730040-00007] [PMID]

[5] Shanmugam J, Kumar PS, Panicker VK, Duvooru P. Sudden death due to giant cell myocarditis: A case report. Cardiol Res. 2015; 6(6):372-5. [DOI:10.14740/cr446e] [PMID] [PMCID]

[6] Matturri L, Del Corno G, Lavezzi AM. Sudden infant death following hexavalent vaccination: A neuropathologic study. Curr Med Chem. 2014; 21(7):941-6. [DOI:10.2174/092986731 13206660289] [PMID]

[7] Osawa M, Nagao R, Kakimoto Y, Kakiuchi Y, Satoh F. Sudden infant death after vaccination: Survey of forensic autopsy files. Am J Forensic Med Pathol. 2019; 40(3):232-7. [DOI:10.1097/PAF.0000000000000494] [PMID]

[8] Choe YJ, Kim JH, Son HJ, Bae GR, Lee DH. Sudden death in the first 2 years of life following immunization in the Republic of Korea. Pediatr Int. 2012; 54(6):905-10. [DOI:10.1111/j.1442200X.2012.03697.x] [PMID]

[9] Naranjo CA, Busto U, Sellers EM, Sandor P, Ruiz I, Roborts EA, et al. A method for estimating the probability of adverse drug reactions. Clin Pharmacol Ther. 1991; 30(2):239-45. [DOI:10.1038/clpt.1981.154] [PMID]

[10] Narasingan SN. Contemporary management of sudden cardiac death. In: Muruganathan A, editor. Medicine Update. $1^{\text {th }}$ ed. India: Jaypee; 2013. http://apiindia.org/wp-content/ uploads/medicine_update_2013/chap31.pdf

[11] Honnekeri BS, Lokhandwala D, Panicker GK, Lokhandwala Y. Sudden cardiac death in India: A growing concern. J Assoc Physicians India. 2014; 62(12):36-40. [PMID]

[12] Sikary AK, Mridha AR, Behera C. Sudden death of a child due to pyogenic bacterial myocarditis. Med Leg J. 2017; 85(2):105-7. [DOI:10.1177/0025817216682187] [PMID]

[13] Kitulwatte ID, Kim PJ, Pollanen MS. Sudden death related myocarditis: A study of 56 cases. Forensic Sci Med Pathol. 2010; 6(1):13-9. [DOI:10.1007/s12024-009-9125-5] [PMID]

[14] Aquila I, Tarzia P, Ricci P, Gratteri S. Sudden death and acute myocarditis: A unique forensic case of double origin of coronary arteries. BMJ Case Rep. 2017; 2017:bcr2017221791. [DOI:10.1136/bcr-2017-221791] [PMID] [PMCID]
[15] Huang WT, Chen RT, Hsu YC, Glasser JW, Rhodes PH. Vaccination and unexplained sudden death risk in Taiwanese infants. Pharmacoepidemiol Drug Saf. 2017; 26(1):17-25. [DOI:10.1002/pds.4141] [PMID]

[16] Brotherton JM, Hull BP, Hayen A, Gidding HF, Burgess MA. Probability of coincident vaccination in the 24 or 48 hours preceding sudden infant death syndrome death in Australia. Pediatrics. 2005; 115(6):e643-6. [DOI:10.1542/peds.2004-2185] [PMID]

[17] Kuhnert R, Schlaud M, Poethko-Müller C, Vennemann M, Fleming P, Blair PS, et al. Reanalyses of case-control studies examining the temporal association between sudden infant death syndrome and vaccination. Vaccine. 2012; 30(13):2349-56. [DOI:10.1016/j.vaccine.2012.01.043] [PMID]

[18] Kumar V, Sidhu N, Roy S, Gaurav K. Myocarditis following diphtheria, whole-cell pertussis, and tetanus toxoid vaccination in a young infant. Ann Pediatr Cardiol. 2018; 11(2):224-6. [DOI:10.4103/apc.APC_107_17] [PMID] [PMCID]

[19] Boccara F, Benhaiem-Sigaux N, Cohen A. Acute myopericarditis after diphtheria, tetanus, and polio vaccination. Chest. 2001; 120(2):671-2. [DOI:10.1378/chest.120.2.671] [PMID]

[20] Wu SJ, Sun S, Li JY, Hu PY, Chien CY. Acute fulminant myocarditis after diphtheria, polio, and tetanus vaccination. Asian Cardiovasc Thorac Ann. 2006; 14(6):e111-2. [DOI:10.11 77/021849230601400627] [PMID]

[21] Geier DA, Geier MR. An evaluation of serious neurological disorders following immunization: A comparison of wholecell pertussis and acellular pertussis vaccines. Brain Dev. 2004; 26(5):296-300. [DOI:10.1016/S0387-7604(03)00169-4]

[22] Fortuna L, Sirivichayakul C, Watanaveeradej V, Soonthornworasiri N, Sitcharungsi R. Adverse events post-DTaP and DTwP vaccination in Thai children. Southeast Asian J Trop Med Public Health. 2015; 46(4):764-74. [PMID]

[23] Rohilla A, Yadav S. Adverse drug reactions: An overview. Int J Pharmacol Res. 2013; 3(1):10-2. [DOI:10.7439/ijpr]

[24] García-Cortés M, Lucena MI, Pachkoria K, Borraz Y, Hidalgo R, Andrade RJ. Evaluation of Naranjo adverse drug reactions probability scale in causality assessment of drug-induced liver injury. Aliment Pharmacol Ther. 2008; 27(9):780-9. [DOI:10.1111/j.1365-2036.2008.03655.x] [PMID] 